\title{
Minimum Variance Gain Nonuniformity Estimation in Infrared Focal Plane Array Sensors
}

\author{
César San-Martin ${ }^{1}$ and Gabriel Hermosilla ${ }^{2}$ \\ 1 Information Processing Laboratory, Department of Electrical Engineering, \\ Universidad de La Frontera. Casilla 54-D Temuco, Chile \\ csmarti@ufro.cl \\ 2 Department of Electrical Eng., Universidad de Chile. Casilla 412-3, Santiago, Chile \\ ghermosi@ing.uchile.cl
}

\begin{abstract}
In this paper, a minimum variance estimator for the gain nonuniformity (NU) in infrared (IR) focal plane array (FPA) imaging system is presented. Recently, we have developed a recursive filter estimator for the offset NU using only the collected scene data, assuming that the offset is a constant in a block of frames where it is estimated. The principal assumption of this scene-based NU correction (NUC) method is that the gain NU is a known constant and does not vary in time. However, in several FPA real systems the gain NU drift is significant. For this reason, in this work we present a gain NU drift estimation based on the offset NU recursive estimation assuming that gain and offset are jointly distributed. The efficacy of this NUC technique is demonstrated by employing several real infrared video se quences.
\end{abstract}

Keywords: Minimum Variance Estimator, Image Sequence Processing, Infrared Focal Plane Arrays, Signal Processing.

\section{Introduction}

It is well known that the NU noise in infrared imaging sensors, which is due to píxel-to-pixel variation in the responses of the detector; degrades the quality of IR images 112. In addition, what is worse is that the NU varies slowly on time, depending on the type of technology that is been used. In order to solve this problem, several scene-based NUC techniques have been developed 34567 8 1 . Scene-based techniques perform the NUC using only the video sequences that are being imaged and not requiring any kind of laboratory calibration technique. In [10] we have developed a recursive filter to estimate the offset of each detector on the FPA. The method is developed using two key assumptions: i) the input irradiance at each detector is a random and uniformly distributed variable in a range that is common in all detectors in FPA; and ii) the FPA technology exhibits important offset non-uniformity with slow temporal drift. The proposed algorithm is developed to operate on one block, short enough to assume that the offset NU can be estimated as a constant in noise

E. Bayro-Corrochano and J.-O. Eklundh (Eds.): CIARP 2009, LNCS 5856, pp. 1047-1053 2009.

(C) Springer-Verlag Berlin Heidelberg 2009 
(ECN) [11. In this paper the gain NU drift is considered and it is obtained from the offset estimated using the ECN method. Afterwards, assuming that the offset and gain are jointly distributed, a minimum variance estimator for the gain can be obtained. This paper is organized as following. In section 2, a review of the ECN NUC method is presented. In Section 3, the gain estimator (GE) proposed is exposed and the results using this method are presented in Section 4. Finally, in Section 5 the conclusions of the paper are summarized.

\section{Estimation of a Constant in Noise NUC Method}

The pixel-to-pixel variation in the detectors' responses is modeled by the commonly used linear model for each pixel on the infrared focal plane array. For the $(i j)^{t h}$ detector, the measured readout signal $y_{i j}$ at a given time $n$, can be expressed as:

$$
y_{i j}(n)=A_{i j}(n) x_{i j}(n)+B_{i j}(n)+v_{i j}(n),
$$

where $A_{i j}(n)$ and $B_{i j}(n)$ are the gain and the offset of the $(i j)^{t h}$ detector respectively and $x_{i j}(n)$ is the real incident IR photon flux collected by the detector. The term $v_{i j}(n)$ is additive electronic noise represented by a zero-mean Gaussian random variable that is statistically independent of noise in other detectors. The ECN method assumes that $A_{i j}(n)$ is a known constant and $B_{i j}(n)$ remains constant in a block of frames, i.e., $B(n)=B(n-1)=B$, and the model of (1) is re-written as:

$$
y(n)=A x(n)+B+v(n),
$$

where the subscript $i j$ is omitted with the understanding that all operations are performed on a pixel by pixel basis. Equation (2) is valid only in a block of frames. The recursive estimator for the offset NU is given by:

$$
\hat{B}(n)=C_{n} \hat{B}(n-1)+K_{n} y(n),
$$

where $\hat{B}(n)$ and $\hat{B}(n-1)$ are the estimates for the offset and $C_{n}$ and $K_{n}$ are the coefficients of the filter. Equation (3) can be recursively calculated using the follows equations:

$$
\begin{aligned}
C_{n} & =\frac{1+a n}{1+(n+1) a}, \\
K_{n} & =\frac{a}{1+(n+1) a},
\end{aligned}
$$

where $a$ is the convergence control parameter typically less than 1. ECN have two parameters, $a$ and the length of the block of frames, $n_{b}$, when the algorithm stops the estimation. The corrected frame is obtained by:

$$
\hat{x}(n)=y(n)-\hat{B}\left(n_{b}\right)
$$




\section{Minimum Variance Gain NU Estimator (GE)}

First of all, it is assumed that the gain $A$ and the offset $B$ are Gaussian random processes and they are jointly distributed. Then, the conditional probability of $A$ given $B$ is defined by:

$$
p_{A \mid B}(A \mid B)=\frac{p_{A, B}(A, B)}{p_{B}(B)},
$$

where, $p_{A, B}(A, B)$ is the joint probability of $A$ and $B$, and $p_{B} B$ is the probability of $B$ (obviously non-zero). After this, a minimum variance estimator for $A$ is formulated from this relationship. With $A$ and $B$ Gaussian random variables jointly distributed, where the information is known with regard to $B$. Then, the estimator for a minimum variance is defined as only a conditional average of $A$ given $B$ :

$$
\hat{A}=E[A \mid B]=\int_{-\infty}^{\infty} A p_{A \mid B}(A \mid B) d A,
$$

where the error $E\left[\|A-\hat{A}\|^{2} \mid B\right]$ is minimal. Then, as $A$ and $B$ are assumed Gaussian random process individual and mutually, they are completely defined by their mean and their variance and $p_{A \mid B}(A \mid B)$ can be expressed as:

$$
p_{A \mid B}(A \mid B)=\frac{\exp \left\{-\frac{\left(A-\left\{\bar{A}+(B-\bar{B}) \sigma_{A B} / \sigma_{B}^{2}\right\}\right)^{2}}{2\left(\sigma_{A}^{2}-\sigma_{A B} \sigma_{B A} / \sigma_{B}^{2}\right)}\right\}}{\sqrt{(2 \pi)}\left|\sigma_{A}^{2}-\sigma_{A B} \sigma_{B A} / \sigma_{B}^{2}\right|^{1 / 2}},
$$

where, $\bar{A}+\frac{\sigma_{A B}}{\sigma_{B}^{2}}(B-\bar{B})$ and $\sigma_{A}^{2}-\sigma_{A B} \sigma_{B A} / \sigma_{B}^{2}$ are the mean and variance respectively. Then, the minimum variance estimator for $A$ is obtained by:

$$
\hat{A}=E[A \mid B]=\int_{-\infty}^{\infty} A p_{A \mid B}(A \mid B) d A=\bar{A}+\frac{\sigma_{A B}}{\sigma_{B}^{2}}(B-\bar{B}) .
$$

In our case, we needed to know that $B, \bar{B}, \sigma_{B}^{2}$ and $\sigma_{A B} . B$ were obtained from ECN method when $n=n_{b}$, the mean and variance of $B$ can be calculated as an approximation to the mean and spatial variance, and the covariance is calculated by:

$$
\sigma_{A B}=R_{0} \sqrt{\sigma_{A}^{2} \sigma_{B}^{2}}
$$

where, $R_{0}$ is the correlation between $A$ and $B$. This index can be measured from other methods of NUC. Finally, using (11) the GE given by (10) is recast resulting in:

$$
\hat{A}=\bar{A}+R_{0} \frac{\sigma_{A}}{\sigma_{B}}(B-\bar{B}),
$$

and the corrected frame is obtained using the following equation :

$$
\hat{x}(n)=\frac{y(n)-\hat{B}\left(n_{b}\right)}{\hat{A}} .
$$




\section{Results}

Real IR video data are used to test the ability of the proposed method and reduce NU. The sequence has been collected at $1 \mathrm{PM}$ using a $128 \times 128 \mathrm{InSb}$ focal plane array cooled camera Amber Model AE-4128 operating in the $3 \mu \mathrm{m}-$ $5 \mu \mathrm{m}$ range. In the data set, 3000 frames were collected at a rate of 30 frames per second, at 16 bits of resolution. There are data of black bodies radiators, which are used to estimate the gain and the offset associated with each detector. With these parameters, the best correction of nonuniformity is performed, obtaining a sequence that is used as a reference.

As a quantitative measure of performance, the Root Mean Square Error (RMSE) was used, which measures the difference between the reference infrared image and the corrected image using the proposed method. The RMSE is calculated by:

$$
R M S E(n)=\sqrt{\frac{1}{p m} \sum_{i=1}^{p} \sum_{j=1}^{m}\left(\hat{x}_{i j}(n)-x_{i j}(n)\right)^{2}},
$$

where, $p \times m$ is the number of detectors in the focal plane array, $\hat{x}_{i j}(n)$, is the infrared irradiance calculated with the gain estimated by the recursive filter, and $x_{i j}(n)$ is the infrared irradiance calculated by the black-body radiator data. A lower value of RMSE means a good correction of the frame data.

Also the roughness index $\rho$ metric is used to measure performance without reference. $\rho$ delivers information about the level of softness that an image has, i.e., the degree of non-uniformity in this image. This index is calculated by:

$$
\rho=\frac{\|h * I\|_{1}+\left\|h^{T} * I\right\|_{1}}{\|I\|_{1}}
$$

where, the image $I$ is the corrupted or compensated frame, a filter $h$ is needed to find the softness of the image, $*$ represents the convolution and \|\|$_{1}$ represents the norm $L_{1}$. In the same form of RMSE a low value of $\rho$ close to zero indicates a good correction.

Initially, we estimate the offset NU using the ECN NUC method. From [10], the value for the parameter $a=0.1$ is the best selection using RMSE and $\rho$. Then, from the estimated offset the gain NU is obtained using (12), and for each value of $n_{b}=\{250,500,750,1000,1250,1500\}$ the RMSE and $\rho$ are calculated and the results are shown in Fig. 1. The estimated gain NU is presented in Fig. 2b and 2d. For all results we are selected $R_{0}=-0.9$ and $\sigma_{A}=0.015$.

Finally, a comparison of the performance method is presented in Fig. 3. In this case, the $1600 t h$ corrupted frame of the real IR sequence is presented (Fig. 3a). The ECN compensates the corrupted frame and the results are shown in Fig. 3b, and the GE NUC method generates the corrected frame in Fig. 3c. The corresponding RMSE and $\rho$ values are presented in Table 1. For this case, the RMSE values are 3.37, 2.96 and 2.12 for the corrupted frame, corrected frame with ENC and corrected frame with GE, respectively. The $\rho$ values correspond to 2.417 for the corrupted frame, 2.108 for the compensated frame using ENC 


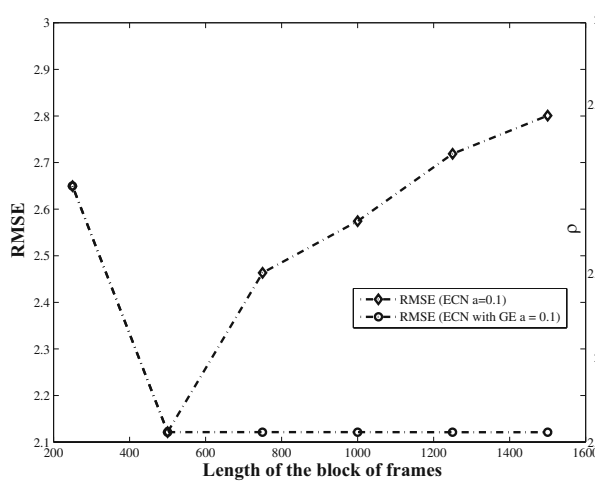

(a)

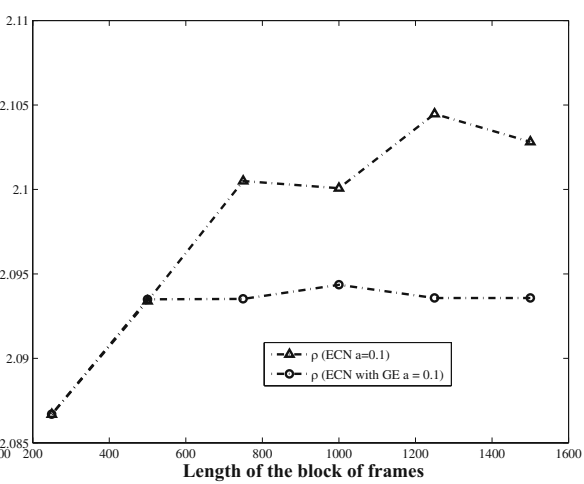

(b)

Fig. 1. RMSE (a) and $\rho$ (b) for the compensated frame using ECN and ECN with GE NUC method. In this case, $n_{b}=\{250,500,750,1000,1250,1500\}$ and $a=0.1$.

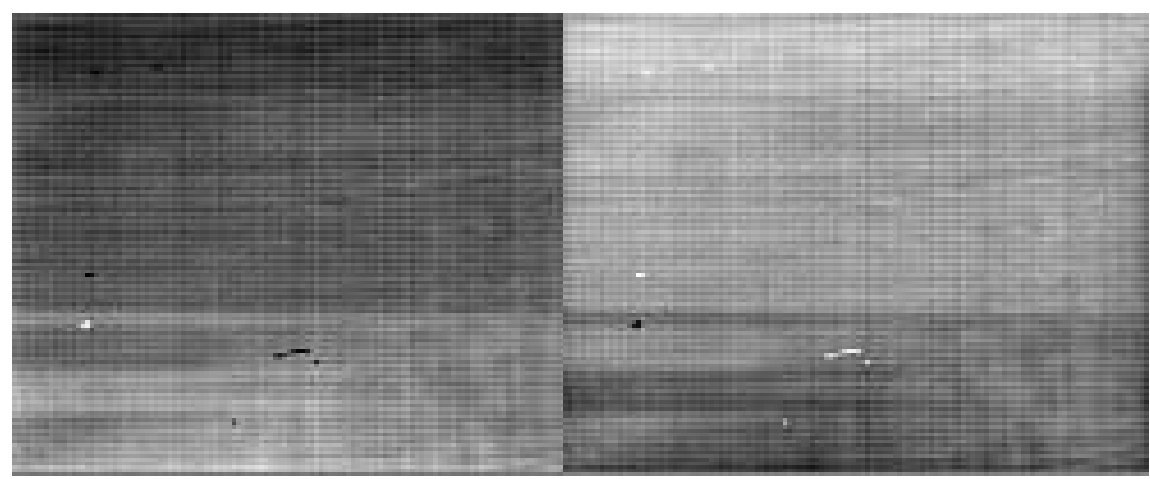

(a)

(b)

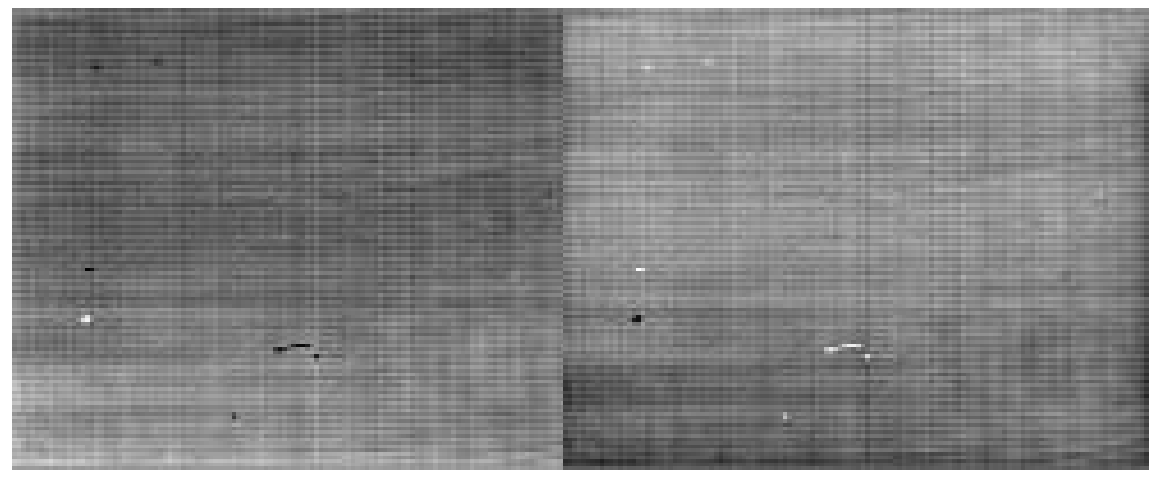

(c)

(d)

Fig. 2. Different offset and gain NU estimated using the proposed method. a) and c) correspond to the estimation of B using ECN for $n_{b}=500$ and $n_{b}=1000$ respectively; b) and d) show the gain NU using GE method for $n_{b}=500$ and $n_{b}=1000$ respectively. 


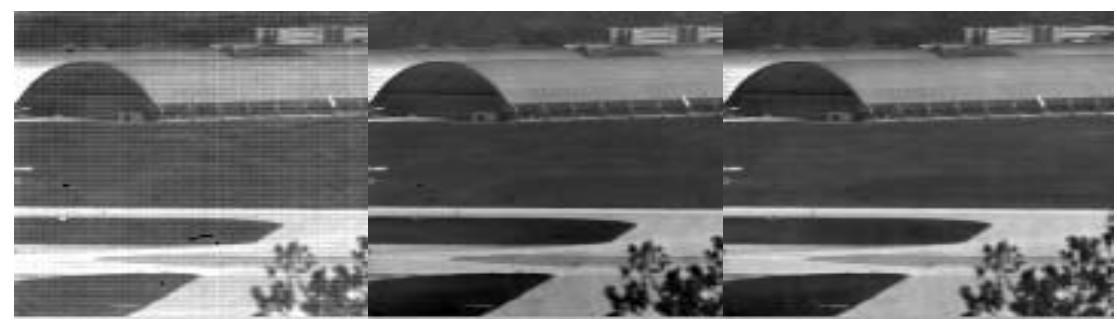

(a)

(b)

(c)

Fig. 3. Performance of the proposed method using real IR data. (a) corrupted frame, (b) compensated frame using ECN method, and (c) corrected frame using ECN with GE method.

Table 1. The calculated RMSE and $\rho$ parameters for real IR frames corrected for NU by using the ECN method and ECN with GE method

\begin{tabular}{|c|c|c|}
\hline Frame & RMSE & $\rho$ \\
\hline Corrupted & 3.37 & 2.417 \\
\hline Corrected using ECN & 2.96 & 2.108 \\
\hline Corrected using ECN with GE & 2.12 & 2.093 \\
\hline
\end{tabular}

and 2.093 for ECN with GE. From these results clearly the proposed method generates a better performance when the gain NU drift is considered.

\section{Conclusions}

A recursive estimation for gain NU on infrared imaging systems is proposed in this paper. It was shown experimentally using real IR data that the method is able to reduce non-uniformity substantially. Indeed, the method has shown an acceptable reduction of nonuniformity after processing only approximately 500 frames. The main advantage of the method is a simplicity using only fundamental estimation theory. The key assumption of the proposed method is that the offset and the gain are jointly distributed. The offset is estimated using a recursive filter, and then, the gain is obtained using a minimum variance estimator. The results presented showed that this assumption is validated with real IR data.

\section{Acknowledgments}

This work was partially supported by Universidad de La Frontera, Proyecto DIUFRO 08-0048. The authors wish to thank Ernest E. Armstrong (OptiMetrics Inc., USA) for collecting the data, and the United States Air Force Research Laboratory, Ohio, USA. 


\section{References}

1. Milton, A., Barone, F., Kruer, M.: Influence of nonuniformity on infrared focal plane array performance. Optical Engineering 24, 855-862 (1985)

2. Mooney, J., Shepherd, F., Ewing, W., Murguia, J., Silverman, J.: Responsivity nonuniformity limited performance of infrared staring cameras. Optical Engineering 28, 1151-1161 (1989)

3. Harris, J., Chiang, Y.: Nonuniformity correction of infrared image sequences using constant statistics constraint. IEEE Trans. on Image Processing 8, 1148-1151 (1999)

4. Hayat, M., Torres, S., Amstrong, E., Cain, S., Yasuda, B.: Statistical algorithm fo nonuniformity correction in focal plane arrays. Applied Optics 38, 773-780 (1999)

5. Averbuch, A., Liron, G., Bobrovsky, B.: Scene based non-uniformity correction in thermal images using Kalman filter. Image and Vision Computing 25, 833-851 (2007)

6. Scribner, D., Sarkady, K., Kruer, M.: Adaptive nonuniformity correction for infrared focal plane arrays using neural networks. In: Proceeding of SPIE, vol. 1541, pp. 100-109 (1991)

7. Scribner, D., Sarkady, K., Kruer, M.: Adaptive retina-like preprocessing for imaging detector arrays. In: Proceeding of the IEEE International Conference on Neural Networks, vol. 3, pp. 1955-1960 (1993)

8. Torres, S., Vera, E., Reeves, R., Sobarzo, S.: Adaptive scene-based nonuniformity correction method for infrared focal plane arrays. In: Proceeding of SPIE, vol. 5076, pp. 130-139 (2003)

9. Torres, S., Hayat, M.: Kalman filtering for adaptive nonuniformity correction in infrared focal plane arrays. The JOSA-A Opt. Soc. of America 20, 470-480 (2003)

10. Martin, C.S., Torres, S., Pezoa, J.E.: Statistical recursive filtering for offset nonuniformity estimation in infrared focal-plane-array sensors, in press Infrared Physics \& Technology (2008)

11. Poor, H.V.: An introduction to signal detection and estimation, 2nd edn. Springer, New York (1998) 